\title{
Interview
}

\section{Expanding DAM into a digital-delivery environment - An interview with Steve Sauder of North Plains}

\section{Steve Sauder}

is the Chief Technical Officer of North Plains Systems Corp., and one of the pioneers of Enterprise Digital Asset Management. Before starting North Plains in 1994, he was senior software engineer for Macintosh software at Crosfield Electronics Inc. While there, he was heavily involved in newspaper satellite wirephoto capture and distribution, experience which proved very useful in the creation of North Plains, and in the design of its flagship product, TeleScope, a distributed software framework for managing very large collections of multimedia data.

\section{ABSTRACT Michael Moon interviews Steve Sauder of North Plains on developments in the market regarding digital value chains, and the evolution of TeleScope.}

Journal of Digital Asset Management (2009) 5, 38-49. doi:10.1057/dam.2008.51

Keywords: version control; metadata; collaboration; XMP; digital value chain; TeleScope; digital asset management

MM: We're here with Steve Sauder - Chief Technical Officer (CTO) of North Plains. Steve, would you give us a little bit of your professional background summary?

SS: Sure. I'm currently the CTO at North Plains. I'm also part of the core team from back in 1994. Before that, I worked in the publishing industry as head of the Macintosh Software Development Group at a company called Crosfield Electronics. Before that I spent some time at Apple. I've been working in this business since about 1985.

MM: Could you give us a brief summary of North Plains and its current business? Maybe a little bit about your markets and customers? SS: Absolutely.

North Plains is, I think, somewhat unique in the market. We have been focused, to a significant degree, on the concepts that surround DAM - even when it wasn't necessarily called 'DAM' in the early stages of the company. Right from its very beginning North Plains has not wavered from that as a unique practice.

In the market, oftentimes, DAM has been viewed as an adjunct to things like marketing automation or enterprise content management.
It has always been our position in the market that there are special requirements for the management of rich media. Specifically as it revolves around the idea of having rich media as part of the creative process. We set out to build a DAM product that fits right into the creative process, rather than creating it just as an archive or a knowledge management tool.

As a result, we've been very successful in deploying large-scale DAM systems at very large, enterprise-class customers. Our largest install also happens to be the largest install in the business, according to Accenture - which is the Warner Bros. implementation. They've currently got somewhere in the vicinity of about 44000 users and several million assets on the system. So it is suffice to say that it's quite a large system and part of their broader, mission-critical infrastructure.

In contrast to other companies, which have started with smaller products and come up, we've done the opposite. We've basically started with these really large-scale deployments. In the last couple of years we've introduced more turnkey solutions - culminating most recently with the successful release of TeleScope 
OnDemand in May - which is a hosted software-as-a-service model for digital asset management.

MM: Steve, we've known each other ...

SS: Forever.

MM: Well, probably from 1996, as I recall. Something around that timeframe. When we did the first DAM tour for Apple.

SS: Yes. A long time.

MM: We've seen the industry evolve through several stages. Initially, DAM was just simple desktop or shared workgroup multimedia databases. The first one out there was called Fetch.

Then came the emergence of the client server/creative workgroup system. That's where North Plains really started to break out - with their Media Manager product.

Then the industry moved into the third wave: media services platforms, with more traditional repository function, multiple user classes and user roles - complex permissions and the ability to dynamically render assets on demand as well as driving a dynamic publishing system or e-commerce application.

We've seen DAM move from the media services platform to now an enterprise contentservices group, where we're not just talking about media assets in terms of rich media assets, but also editorial assets or textural assets and XML databases.

Finally, we've seen the emergence in these last few years of the notion of digital supply chains. We now have multiple business entities whose systems connect. I guess the technical term is a 'loosely coupled system,' which is part of an end-to-end process. Each individual system remains autonomous and operates on its own set of governance and policies - but plays in terms of a larger federal governance and process control structure.

With that as a story, can you add anything to that or clarify any of the key points of that particular line of development?

SS: You've done a very good job of summarizing the entire DAM market.

I think from North Plains' perspective, when we first came into the market, there was a vision of DAM being an archival system. It was used basically to 'not lose assets' and to repurpose them. We saw that as an initial step - and certainly an important process point for the DAM market.
But we came into the market thinking that if you just used DAM as an archive you were really only half-using the product and therefore only getting half the benefits.

We saw DAM as a work-in-progress tool, as well. That fed into a lot of our initial decisions on how TeleScope works. We have the hybrid file system, which basically allows us to act both as a repository, but also to provide managed access to the assets, through to other creative applications like InDesign, PhotoShop, Quark and so forth. Now, we're seeing more demand for video functionality so North Plains has focused on developing applications that support Final Cut Pro and Avid.

By including the DAM system as part of the creative process, we found that it made metadata gathering much easier. Metadata is a hugely important issue in DAM. When you're looking at managing e-mails or documents or any of the more textual-based assets, there's human readable content there that can be leveraged.

But such things didn't exist or still don't exist in a lot of cases for rich media content - at least until the advent of some of the metadata standards like XMP. So how you tag and keyword assets becomes really important for any and all future search activities.

There's this barrier that exists where someone who's not involved in production - the librarian has to figure out what each asset really is, and how to tag it appropriately before putting it into the DAM. That process becomes heinous especially as you start working with large volumes of content.

By embedding the DAM system in the workflow process, the fact that it's used in a particular publication or the fact that you have a review-and-approval cycle and users leave comments about what they think of the assets those sorts of activities and input all become part of the searchable metadata ecosystem that exists around the asset as it works its way through production.

By the time you are ready to finish and print the book or publish the content on the web, there is sufficient metadata that you can probably find it again later without even having to look at keywording as a specific task. This can save a tremendous amount of search time. 
It's really about building the metadata and building the asset as an entity that works its way through production.

What's been interesting is that lately that process continues to be extended further into an organization's workflow. You talked about digital supply chains. That's really a huge thing. It gives us the next step in that logical progress.

We focused initially - as I think a lot of DAM companies have come to focus, as well, on the product side of things. How to save money creating these automation and workflow management processes. In addition though, we've included enhanced functionality on how to store and manage assets while simplifying how to repurpose and reuse them later.

Then there's a whole added piece on the end of that lifecycle, which really hasn't been managed up until this point. And that's the monetization of the content.

There are whole middleware layers that are appearing in the market and have been appearing for the last little while. Things like The Platform and these rich media distribution platforms that are there, basically to take content, monetize it and finish that product lifecycle.

We've been working pretty extensively on that over the last 6 months, and looking at where DAM moves after the production side of things is finished.

MM:You brought up a couple of issues I want to have you further expand upon.

One of the things that generally many people in the industry recognize and associate with North Plains is that because it came from a creative production environment and has a very rich, robust, stable, fat client that sits on Macs and PCs and so on ... It really synced up well with the creative mindset.

In a production environment, creative people get really annoyed with anything but sub-second response time for everything on their desktop. Having them move in and out of browsers tends to be a tedious, annoying experience for many creatives. It seems to me that that was one of the areas of strength for North Plains TeleScope.

Could you expand upon what it takes to really support the creative team - specifically around this work in process? SS: Well, it's a good point. It continues to be something that I believe we excel at. A lot of the more recent accounts that we have won have been won because we can demonstrate an understanding of the process.

Let's face it. It takes a couple of minutes to write an e-mail, but it takes on the order of a couple of days or maybe even a couple of months to create a properly edited professional video. So the amount of work and the cost of that work that goes into the creation of this rich media content is orders of magnitude more than what it takes to create an e-mail or a document or some of the more traditional things that content-management systems manage.

So, the support of the production workflow through the DAM system is vitally important. As I mentioned before, there are design decisions we made with TeleScope at the beginning of the process that have supported that. The ability to do this managed file sharing, so that TeleScope assets can appear as real files on real file systems, with real server access to applications that need them - as opposed to the traditional repository model, where if I want to use an asset in production, I have to check it out of the DAM system onto my desktop, use it and then possibly check it back in when I'm done with it.

That leads to multiple unmanaged copies of these assets sitting around on peoples' desktops during production. That's really the antithesis of what you're trying to accomplish - which is control over the content. That idea of the managed file system becomes really important.

MM:You have a Photoshop, Illustrator and InDesign files or video file, and that physically lives in a managed file system.

SS: That's correct.

MM: As a creative individual who then wants to somehow modify, edit, enhance that particular file from my desktop application, I will open that file across the network. As I save changes, I save them back across the network to the physical file in your managed file system, as opposed to storing local copies. Did I understand that right?

SS: Correct again. You can, in fact, use TeleScope's Version Control System to check out a local copy that you're working on. Then when you check it back in, it goes back into our managed file system. But for a traditional publishing workflow - for example - I'd have my InDesign page open on my desktop. Using TeleScope, I can drag a thumbnail out of the 
TeleScope environment and drop it onto the InDesign page.

TeleScope passes to InDesign a computed pointer to that file that's actually pointing to it in our repository. But it's manufactured in such a way that it appears to InDesign like a standard file pointer on a server. We basically reproduce that illusory file server for them on the desktop. That stems from two concepts.

One is, InDesign doesn't know about DAM repositories. It knows about files and folders. So doing it this way means I have the capability within TeleScope to work with a vastly larger number of applications. Otherwise I'd have to start writing custom plug-ins for InDesign and Quark and all of those things, to make those applications understand TeleScope's environment. That's something that the document management systems, for example, have done for years. But it's a lot of work and not logical.

Doing it this way, TeleScope is able to securely represent files as though they existed on an ordinary file server, when in fact they're being managed and monitored within our file system.

So then the other benefit that it has is, if I take an InDesign file and put it into TeleScope and I download it and open it on somebody else's machine, the links will not be broken. This is because the links are pointing to the files on our server and not pointing to some copy of the file sitting on the designer's desktop.

It's a way of not only preserving the security of the assets themselves, it's also a way of managing the workgroup work-in-progress functionality.

That becomes especially important with video. If I have a half-hour long MPEG2 broadcast video sitting on my XSan - the last thing you want to do is begin migrating it around the network, from desktop to desktop, as you want to work with it.

We found that that model, which we had originally created for the publishing market, actually in fact makes TeleScope incredibly useful for video editing. I can basically take a thumbnail of a video clip in TeleScope and drag-and-drop it onto the timeline in Final Cut Pro. All I'm doing is passing Final Cut Pro a pointer to that video asset on the server. It becomes really useful for most video editing workflows.
MM: That represents a relatively new development for TeleScope - handling large motion graphic video and animations and so on. SS: Huge. Yes.

MM: Could you take us through or explain the process of ingestion, specifically for video?

SS: It was something that we spent a great deal of time working through with customers. And also with prospects that were kind enough to take a flyer on what we were looking at doing. There really were no video asset management systems out there at the time we were looking at this that did what they wanted to do - at the scale they wanted to do them with.

We had the benefit of working with some of the best and brightest in the business. Like Viacom and TV Ontario, here in Toronto. You can't put a price tag on that kind of experience.

It's got a lot to do with the base problem. In still motion or document functionality in the DAM system, you have a piece of metadata that's associated with the asset itself. Although you still need that for video, the thing that's really tricky about video is that what's true $1 \mathrm{~min}$ in the video may no longer be true $5 \mathrm{~min}$ later or even a few seconds after that. Right? MM: With each discrete scene, you have a new thing to catalog.

SS: Exactly. Especially when you're looking at trying to accurately represent what is being said, for example. Things like speech-to-text. You really need to get to the level where you're able to add metadata to an individual frame in the video. That's where we started to go with this idea of time-based metadata.

With the introduction of video asset management capabilities, video became a real first-class citizen in the DAM world. The first thing we had to do was to have the ability to be able to actually annotate and put metadata on each frame. The time-based metadata capabilities in TeleScope Video Manager gave us that capability. That was a whole new structure we had to build into the platform.

But even more interesting is - as an example if I take a TIFF file that's high-resolution and I put it into the system, TeleScope has traditionally operated in what we call the 'Digital Master Model.' That's basically where you take the highest quality, best resolution version of the file you're working on, and you put it into the DAM system. Then if you need it an another 
resolution or for web use or for use in your Power Point, you can let the DAM perform that translation for you.

So we built file conversion capabilities right into the application.

But what's interesting is that for video, file conversion is an extremely expensive process. Transcoding video from high-resolution MPEG2 to low-resolution Windows Media, for example, could take up to half an hour to complete. Asking the user to wait for that while they're actually doing the downloads is problematic.

Instead, for video, we need to support the idea of pre-conversion. What that means is that when you ingest a video, TeleScope Video Manager is able to automate the process of creating low-resolution proxies of the videos. We work with a number of class-leading transcoding solutions so we have a system that can be used for any content type.

For example, for web-based content, I might generate a Flash proxy. For broadcast, I may have an HD and an SD proxy in the appropriate formats for whatever a customer's playout servers are. It's a method of pre-creating what you want as your output format.

In addition, we can also generate lower resolution proxies that are suitable for previewing. The last thing you want to do is play 16 gigabytes of video across your network just to be able to put an annotation on a particular frame. The concept of utilizing preconversions specifically for playback was something that we introduced with Video Manager, as well.

We took advantage of a structure that was already available in TeleScope, called 'Renditions.' It basically allows a single asset to be managed and manipulated simultaneously to create 'different flavors' of the original 'video essence.' MM: If I understand you right, here I've got a digital beta or a high-resolution source file. Half an hour in length. As I ingest it and upload and put it into the TeleScope DAM, you specify what your pre-renders are.

SS: Yes.

MM: Upon completion of the ingestion process, there could be a pre-rendered Flash file or a QuickTime file or a Windows Media file that's pretty much an exact copy of the long form piece that I put in.
SS: Right. Although it might be at a much lower resolution, it might be a lower bit rate. It might be a different pixel dimensions. For example, I might size it down so that it's not as large.

MM: So then as I make time-based edit decision lists (EDLs), and as I start editing by using an offline editor, it puts together a decision list, and then I say, 'Okay. Go give me that.' It could just simply go and get that as an edited, compiled Flash stream from the pre-existing Flash file, as opposed to going back to the high-resolution file and having to re-render it all down.

Did I understand that right?

SS: That's a good succinct overview of the way that it works. Although, there's one thing that is generally different.

When you talk about editing you're talking about using Final Cut Pro and those sorts of applications, which generally is the goal. But we're finding what's happening now in video is the same thing that happened a few years ago in photography. That is, that the switch to digital has caused there to be a glut of content that needs to be managed.

Now that it doesn't cost anything to shoot more, there is a significant jump in shooting ratios, in fact multiple times the amount of raw content for the same amount of final edited video.

MM: In fact, as we've talked to Playboy and Victoria's Secret and some of these other very visually driven brands - and the conversion from analogue camera backs to digital camera backs, they're seeing a 10- to 20-time increase in terms of the number of images produced on a shoot. SS: Yes. It's even worse for video. We've seen shooting ratios go from 5:1 to $10: 1$, which is standard film. You didn't normally shoot 5-times coverage.

MM: That's for straight-forward documentary content. Right?

SS: Right.

MM: For feature films, it usually is 100:1 or 200:1. Right?

SS: Right.

But even in that environment, the worst is generally commercials. They'll generally shoot 3 or 4 hours' worth of content for 30-second spots. That's a 300 to 400:1 shooting ratio.

Lars Von Trier, for example, as a filmmaker, is famous for mounting 100 digital video cameras 
all over the place on his sets. He'll basically let them all run and then sort out the mess in the editing suite. So we're seeing 1000:1 plus shooting ratios.

One of the worst is reality television. In reality TV, they basically leave the cameras running $24 \times 7$. Then once a week, they've got how many hundreds or thousands of hours worth of content that they have to narrow down to a 30- or 60-min show.

That's a huge glut of excess video to review. Who's paying for this? Editors. They're spending far more time in the edit suites and far less time actually editing. They're just scrolling through all this stuff - most of which will never be used. Traditionally, it's stuff that would be left on the cutting room floor.

We coined this phrase at NAB 2007 - the 'crap-to-content ratio.' The crap-to-content ratio is really getting very high in video now. Traditionally, in photography, photographers turned to digital asset management to build workflows that help manage that glut of content.

You mentioned Playboy. That's certainly one of the customers we've been working with on defining this process.

You essentially need to define a new job description in the standard editing workflow. The editor is swamped with too much stuff. You need someone between the camera and the editor. They need to be a producer on one hand because they need to know what the end result is supposed to be. But on the other hand, they need to know what makes a good cut and where a good scene change would make sense: that kind of stuff, so they can start looking at rough cut editing of the content.

This combination of 'producer' and 'editor,' we started calling 'preditors.'

MM: That would actually be pre-editor. Right? SS: Exactly.

They basically precut the video content so that what gets sent through to the actual edit suites in order to generate the final content is pared down to what's interesting.

MM: That pre-editor or preditor function is generally done in-field?

SS: It can be done in-field. In a lot of places it's done back at the post-house. But it's generally not done in the edit suite, specifically.

MM: The edit suite's running $\$ 500$ to $\$ 1000$ an hour type of thing?
SS: Exactly.

What we've been proposing to a number of customers who are having this problem is to use the DAM system as that tool. You ingest the content and use the low-resolution proxies, which are much easier to work with. You go through, using the Video Manager tool within TeleScope. We've added in the capability for very easy 'mark-in' 'mark-out' clip creation.

In fact, within Video Manager, I can take those clips that I've created and I can arrange them in a timeline within the TeleScope application, and essentially preview a rough cut within the TeleScope application. It's not perfect. The cuts may be 2 or $3 \mathrm{~s}$ off where they finally end up. Depending upon your proxy, it might not necessarily be frame-accurate. But it is good enough to be able to say, 'Okay. This is in general what I'm looking for.' Then we can generate the EDLs that actually get sent to Final Cut Pro or Avid.

The actual craft edit is being done based on only the information that's interesting to the organization. It's a huge time-savings and money-savings, too, because of course you pay the preditors less than you'd pay your professional editor.

MM: Could you bring us through that same process, describing the creation of metadata and other sorts of contextual information?

SS: Right. Metadata becomes important in these cases depending on what you're doing. For a video production company, oftentimes when you're sitting in the production environment, you're at-camera. They'll put some metadata in and associate it with the SMPTE (Society of Motion Picture and Television Engineers) codes that they're seeing on the tape. That information is either unfortunately still written down or stored in a FileMaker Pro database or put into an Excel spreadsheet. Something like that.

MM: This would be essentially - to use a paperbased metaphor - the 'job jacket?'

SS: Exactly. Except that it's associated with time code. That way you know where you are in the overall scheme of things. We need to be able to pull that in. We've done integrations with products like Pilot Ware, which has been pretty heavily used in the reality TV arena and others. To be able to pull this time-based metadata into TeleScope, and start using that as a basis for doing some of these pre-edits. 
Then there is content that comes from professional sources to begin with. We have a lot of customers who use TeleScope for this too for example - educational institutions like the Utah Education Network (UEN). It uses TeleScope to provide PBS and other educational content to K12 education across the entire state of Utah. They make heavy use of the clipping and metadata functions within TeleScope. The teachers themselves are logging in and saying, 'Okay. I want to find information about whatever it is I'm teaching tomorrow.'

They'll pull up 30 or 40 clips and be able to pull individual sections out of those, all on the fly, generate a video that they can play in the classroom the very next day. For those things, the time-based metadata becomes really important.

Gathering the closed-caption information automatically out of the content and associating that with time code is also critical. As teachers generate their own clips, they can leave markers and annotations behind to say, 'Here's a really good clip about this,' or something like that. And other educators - as they go along - can quickly pull those things up for their own needs.

It's a user-generated content process, where at particular sections of the video, you can leave markers for other people to find. Our customers have found it to be very useful.

MM: It reminds me of a conversation that you and I had at one of the symposia. You were describing the creative use of sticky notes digital sticky notes - as a way of creating ... to use a somewhat controversial term, a folksonomy of informal tags. That was part of the overall search. Part of what would help facilitate a more target search.

SS: Yes.

MM: Could you bring us up-to-speed on that? SS: We started out with the idea of being able to add notes. It was just basically a glorified metadata field where you could just add notes about an asset. In the case of video, you could add notes about a particular frame of the asset.

They just became part of a searchable metadata structure that was there. We were doing that some time ago. All of a sudden when YouTube came out, it became apparent that if you actually relied solely on the description supplied by the person that uploaded a video as the searchable data, you'd never find anything, because people don't typically supply much (if anything) in this data.

YouTube very quickly and cleverly found out that what is more important are other users' comments that occur over time. All somebody has to say is, 'Oh, I hate Michael Jackson,' and poof - it's a Michael Jackson video. I think this is where the idea of folksonomies came about ... When you're searching on these commentaries, you're actually searching on information that's not really designed as metadata. It's not accurate.

So, consequently, you have to search it in fuzzy ways. We took that concept and applied it to the idea of sticky notes, and from that, we came up with the idea of discussions. So every asset in TeleScope becomes a discussion forum. People can respond to each other and everything is date- and user-stamped. There are moderators who can remove things and remove offensive entries and all that stuff. It's all really easy to manage.

As we were building it, I was thinking, 'But this isn't business.' Right? People typically don't use in-house DAM systems in the same way as YouTube. If I'm going to leave a message, it's because it's important, there is much less that is 'frivolous.'

What we found was that it has become really useful for things like review-and-approval cycles. If I have to review an asset and I've got a bunch of people that are in my creative team and we all have to review a particular thing and decide whether we like the color or whatever ... That's part of the standard review process. This may extend outside the organization to customers, for example. For ad agencies, that's quite important.

Being able to preserve and save these comments - basically, a transcription, like what we're doing with this interview - that review process really adds value to the metadata that's associated with the asset.

We're actually taking that to the next step. We're releasing a new product that's in development now called TeleScope Live Collaboration. It allows each asset to become a 'meeting room' where not only can I put textual markup on there, but I can actually do graphical markup. I can draw circles. I can leave lines. I can position sticky notes spatially, like real sticky notes. 
We can scrub all the text off of that and make it searchable. But it's also graphical markup. For documents, I can do that on individual pages. For videos, I can do that on individual frames. It's the same basic concept.

But what's neat about the live collaboration product is that I may have multiple users sitting there looking at this asset at the same time, and they will all see the same view. It's VoIP-enabled and supports webcams, so I can see little video thumbnails of the people from their webcams, and it becomes a real-time discussion forum where I can take the talking stick and I can take a document and flip to a particular page and on everyone's screen, it flips to that page virtually.

Then I can draw a circle around a word and everyone will see that circle. It becomes a much more interactive process that way.

MM: This requires that each of the users have a TeleScope client? Or are you doing this through a browser?

SS: Both. You'll be able to do it through your browser as well.

MM: Fabulous.

SS: It's a pretty neat technology. Actually, I did a sneak preview of it at our TeleScope User Group Conference this year and the feedback was exceptional.

MM: Excellent.

As we kind of get close to the end of our conversation here, Steve - one of the things that I thought you could probably bring us up-tospeed on are some of the developing standards around metadata. You mentioned XMP. Are there any other developments in metadata as it relates to specifically the creative workflow and then generally the enterprise or digital supply chain? SS: TeleScope has always been in this space. One of the key components of the way TeleScope works is because we're deployed in so many different markets, a 'standard' metadata model for publishing, for example, would be much different than a standard metadata model for video. TeleScope has always had this interesting concept with its dictionary-based data model.

You can have an infinite variety of metadata models in TeleScope. That's always put us in a kind of sticky situation. If you want to transfer that metadata model to some other component or some other system, there was really no standardized way of representing that. So we had to come up with our own - which we call
MIMiX (it stands for Metadata Interchange Model in XML). It's a TeleScope concept, although I've seen it used in other applications usually as a part of integrating with TeleScope. MM: Could you take a moment and just kind of reprise us of MIMIX?

SS: MIMiX is an XML structure that doesn't impose any semantic preconceptions on what it contains. The problem with most metadata 'standards,' for example Dublin Core, is that they impose a specific set of semantics on their structure: they basically say: 'I have a country field. The country field is interpreted in the following way, and here are some of the valid values.'

The problem is that if I'm an ad agency, I might need that country field. If I'm a publisher, I might not. But I may need something else that's not included in the standard, and there's no way to put that in there so long as there's a semantic assumption in the way that the standard is defined.

MIMiX essentially defines what we call 'meta-metadata.' This means that the MIMiX structure incorporates not only the data, but also information about the data. What its name is, what its data type is, what it represents, how it's used and so on. All of that is explicitly incorporated into the data model.

Instead of saying, 'Here's the country field, and here's what its value is,' the MIMiX structure would say, 'Here is a field. It's a character field. It's 32 characters long. It is interpreted as a country and it's used in the following ways. And here's some alternate terms are for it,' those sorts of things.

It's basically a description not only of the data, but also of how the data are to be used. MM: As I recall, you summarized this back when you introduced it 5 or so years ago. Maybe even longer. You described the insight out of which MIMiX came. A metadata field is actually just another type of data.

SS: Right. That's a good way of putting it. I don't even remember saying that. You've got a great memory, Michael.

MM: You know, when you go into any particular organization - especially larger ones from a Disney to a Warner Brothers to a McGraw Hill - typically, you'll find 5, 10 or 15 unique DAM systems. And each of them has their own metadata schema.

SS: Right. 
MM: In one schema, they might call this person an author, and in another one, they might call him a creator. In another one, they might call him a writer. Referring to essentially the same person, but with different metadata field descriptors.

SS: True.

MM: Then when you want to start moving assets from one system to another to preserve the metadata, it really becomes a challenge. I had one CTO refer to it as 'the ultimate rat hole in the universe.' In terms of how you'd synchronize metadata across these rather large content and digital asset management systems.

SS: Absolutely. The purpose for MIMiX's introduction was because - in our case - that can happen even between TeleScope implementations. Because the system supplies complete flexibility in the metadata model on an installation-by-installation basis, that issue can exist even between two separate TeleScopes.

In fact, they may be running in the same organization. We've run into that in numerous cases. We have several organizations where there's a central enterprise repository and a separate repository for a sub-business that perhaps may have been acquired or whatever. Also using TeleScope but with a different data model. And we need to be able to figure out how to move metadata between those two systems.

MM: So, MIMIX allows you to do some kind of tactical synchronization between two or more disparate systems.

SS: Right. MIMiX, and a TeleScope component called 'XML Gateway.' It allows us to push MIMiX-formatted metadata between two separate systems.

MM: Right. Doesn't that then follow that we'll see the emergence of specialized metadata databases? Where specifically, the data in the database are the schema values.

SS: That seems reasonable.

MM: And that a simple query ... I'm getting carried away, here. But if a simple query on this database could generate - ad hoc - a new schema?

SS: Okay. I think, if I'm following you ... MM: That is to say I could model a new business process.

SS: Yes.

MM: Because the metadata are the lowest common or the most molecular expressions of business rules and logic. Yes?
SS: Right. Yes.

MM: So at the end of the day, any time you start to change a business process, a workflow... ultimately somebody has to rejigger the policies. Rewrite, edit and modify business rules to support those new workflows. Right?

SS: Right.

MM: As we keep moving backward or down into the real DNA of the organization, you could say that a policy at the lowest molecular level is a set of metadata in a schema.

SS:Yes. That makes sense.

MM: So does it then follow that you can facilitate organizational transformation at the level of business process and business model as a function of having agile, fluid metadata in a metadata database that would say, 'Okay. From this point forward - in order to monetize this particular opportunity - here's the metadata that you'll need in order to facilitate that particular set of transactions.'

SS: Yes. To a certain degree, that's something we've built into the TeleScope environment. You're able to revise the metadata model and therefore change the MIMiX structure at any time in our dictionary-based data model without negatively impacting the system. Customers don't have to 'get it right the first time.'

TeleScope is obviously still focused on the data itself. Not necessarily managing the models. But I see where you're going with this. MM:Yes. Here's one of the big insights that we've had this year or so. Traditionally - moving out of the media and entertainment industry and just in general, in terms of the marketing organization or marketing operations ...

Traditionally, the marketing organization has had the job of putting lipstick on pigs. Dressing up what my product manager and my product groups gave me to sell. Seth Godin wrote a book about it called, 'The Meatball Sundae.'

It's the old story of marketing and sales has to sell what you've got - irrespective of whether it matches or meets market requirements and customer criteria for value and satisfaction. SS: Yes.

MM: As more and more of the marketing has shifted to an online and interactive mode - as more and more of the consumers start using interactive modes of relating as a way of determining who gets my business ... Marketing organizations are now in the business not only 
of telling stories - but they're now in the business of provisioning IT applications in the form of customer self services and engagement interactions with the brand and the buying process.

SS: Right.

MM: At some point, you've got to think through that a lot of those applications entail a lot of rich media. Rich media that might not necessarily exist in usable fungible form, unless you've got a rich media repository and the ability to provision them in ways that were never originally envisioned. Specifically, embedded in these various Flash or Air or ServerLITE types of applications.

SS: Right. That whole concept leads really nicely into a specific market - into what we're calling the TeleScope Publishing Platform.

Having tried with a modicum of success over the last number of years to come up with a 'nonstandard standard for metadata' basically, I think it's an extensible idea. We finally had to give in and say, 'For different markets, there are different standards. And we're going to essentially be forced to adhere to whatever those standards are.'

In publishing there's the ONIX standard. This standard revolves around the idea of ISBNs, books and authors and all of that kind of stuff.

What's interesting is that the actual books themselves represent marketing objects for the publishers. Looking at the way the digital asset management fits into the publishing workflow, we've traditionally been mostly involved in the production side of things. But, tying into one of the things we discussed before - which is looking at the digital value chain for this content, through its entire lifecycle - where does DAM end?

If you look at what a publisher actually does, they publish books - so if you focus on the production process, then when you've gotten the printed book published, does the DAM's job finish? Traditionally, you might say yes.

Now, we're starting to say no. We have this content available in our system. If you go to Amazon.com right now and look for a book you like, chances are relatively good - depending upon the publisher of the book - that you will find a 'browse inside' icon where you can start reading individual pages of the book.

That's a very cool feature of Amazon. They've proven empirically that it increases sales and buy-through for that particular book. It, for all intents and purposes, replicates the bookstore experience where you can go in and browse for something you're interested in.

MM: Yes. Browsing the book and reading the dust jacket. Yes.

SS: So by and large, publishers think this is a great thing. Except they don't like the idea of having to give up their intellectual property to Amazon to be able to make it available to the consumer.

We see a huge need in the market right now to actually be able to take content that we in fact already have in the DAM as part of the creative process, and be able to turn that into marketing content. Being able to make the metadata and all the other information that's important there - plus the content itself - be made available through the word you used ... 'Lightweight.' Low-cost functionality.

The idea of Flash 'browse inside' widgets and things like that are in the service of that need. But you need to be able to track very carefully who's viewing what and for how long and so on. So you can find out for marketing purposes what the benefit is of having this stuff available out there. Right?

The whole publishing platform concept is something that we're mapping out very carefully. We're taking the content and pulling it out of the DAM sphere and moving it into the Internet sphere. Making it available through a Flash widget that people can embed on their blogs, for example; or that we can pass through to Amazon; or allow Google to search through and so on ... We're making it part of the larger Internet realm, instead of just archiving the content for the publishers.

MM: Fabulous.

SS: Yes. It's quite interesting. There are a bunch of large companies that have been looking at this concept, like Ingram, who approached publishers and said: 'Hey. We're willing to digitize all of your content for you and make it available for searching and browsing on the Internet. All we ask is that you give us your intellectual property.'

And the publishers have generally responded: 'Umm... I don't think so.'

Companies like LibreDigital have built a business providing these services as a digital warehouse. Of course, once everybody 
standardizes on whatever the format is they want to have for e-books, it's only going to get more interesting.

MM: This would be a good coda for our next conversation. That is the evolution of the book. Just to reprise one item that you introduced, here - this notion of Ingram.

As I recall, they had a strategic initiative about 2 years or maybe 3 years ago. They said, 'We're going to build a big publishing platform targeting small- to mid-sized publishers who can't afford this digital infrastructure.' Essentially, we'll not only distribute the hardcopy book that you print and send to us and that we warehouse ... but that you'd send to us the digital assets from which the book was made.

We'll house it in our large publishing repository as a digital asset, still in its fluid, unformed state. We can create e-books, podcasts, slice-and-dice it - and thereby create incremental revenue streams. Harnessing all this digital technology in ways that you can envision but can't afford because you're a small- to medium-sized publisher.

Did I summarize what Ingram was trying to do?

SS: Absolutely. You summarized it perfectly. And you know what happened, right?

The publishers said, 'No way.' It amazes me that, in proposing this concept, there was a complete failure to realize the inherent conflictof-interest in the distributor controlling content that they had no hand in creating.

Call them paranoid, if you will, but many publishers' first reaction was: 'You are trying to undermine my relationship with the author.' And Ingram had no answer to that. It's the same concern publishers have with Amazon.

One of the reasons you find that large numbers of books on Amazon still don't have the 'browse inside' functionality, despite all their efforts to market it as a service to publishers. The publishers just don't want to give Amazon their content carte-blanche. It's an interesting thing to watch happen.

We believe that for publishers, who are already entrusting DAM systems with the safekeeping of their production content, DAM companies make a lot of sense as a "neutral third party' for turning that content into online browsable and searchable forms, and making that content available globally.
MM: Steve, this really introduces a concept we've been developing now for several years. It's the notion of a center of excellence.

In a center of excellence, I describe abstractly or generally as an autonomous business unit with a profit-and-loss mentality. It invests and continues to invest capital and/or profit in ongoing process improvement, efficiency and essentially automation.

It has some sort of management processcontrol framework. But there's some kind of closed loop, statistical process quality control improvement methodology that's driving the ongoing cost-reduction and cycle-time reductions and quality improvements.

Finally, this center of excellence will do just one thing brilliantly as opposed to many things okay. We've seen the emergence of centers of excellence for pan-regional collateral operations. They do one thing.

'We localize.' We take an English design master, and - using a highly automated collateral factor - translate or localize that into 25 languages appropriate for Europe, Middle East and Africa. And we do that at a third of the cost, a fifth of the time that other means will afford.

We've seen that same thing happens in content operations or digital asset operations, where they become focused on, 'We own, control and maintain governance and policy around all of the ingestion, quality-control and taxonomy and metadata development and how we add new items to our 40 keyword list and so on.'

It seems to me that you've simply called attention to the fact that there is a new center of excellence that's likely to emerge around what we'd call 'publishing operations.' Publishing operations is basically DAM on steroids. But we're also then talking about things like content optimization. There, we have text, text mining, XML repositories that are all part of the overall factory.

SS: Yes. Conversion to e-book formats. Those sorts of things.

MM: Not just convert into e-book format, but really to facilitate what I might call the radical transformation of learning and of reading.

I think books are going to be around for a long time, but that we're going to see some very novel, innovative ways of conveying information, 
knowledge and cultural heritage. Largely, those new expressions will reflect the sensibilities.

As they have a much more tribal, peer-topeer set of social norms, consumption of information and consumption and formation of knowledge will also undergo a similar transformation to reflect those new sensibilities. SS: Yes. That's already happening in book publishing. We're getting a lot of interest from book publishers in allowing readers to create 'mashups' of book content. They'll want to borrow a chapter from this book and borrow a chapter from that book, and then build their own books on the fly that contain the specific information that they're interested in.

It's an extension of the model that I was talking about for UEN, where teachers can pick a scene from here and a scene from there, and put it together into their own video that will be tailored to a particular classroom curriculum.

MM: One of the things we've seen in that concept, Steve, is not just taking professionally generated content, but also taking pro-sumer or user-generated content in the form of blog posts. And threaded discussions in forums. Taking that and melding that back into the printed.

Quite literally, there's a chapter from a college textbook and then there's a whole discussion thread related to the subject of the topic. SS: Yes.

MM: That's, I think, really the next frontier. This integration of generated-generated, embargoed, policy-managed, rights-managed content. Plus all of this other open-frontier stuff that's really user-generated. It's oftentimes a new way of bringing into focus and enhancing the overall engagement potential of the professionally generated content.

Does that make sense?

SS: Absolutely.

MM: So, I think we've definitely gotten to the end of our time, here. Any last summarizing comments or forward-looking things in terms of what you anticipate over the next year or two? SS: I just think this is a really exciting time for DAM in general, and certainly North Plains. We're finally breaking the 'archive' model, and looking at how DAM feeds real business processes and real marketing capabilities. DAM is becoming something that isn't just necessarily for high-end creative processes anymore. All of a sudden, perhaps it's the results of DAM that are interesting.

Perhaps, it was only a matter of time before this started to happen. Speaking as a content creator, now that I have solved the most pressing of the creative, production and repurposing available to me instantly, how am I going to use it - repurpose it - monetize it? That involves really getting into the world at large. DAM is no longer dealing only with the needs of creative users - photographers, designers and so on and we now have to start looking at what does a standard book reader want to see in their widget when they're flipping the pages of the book online?

The market is becoming really interesting. We're expanding and DAM is expanding outside the roles that it has traditionally held.

One of the things that I think is pertinent is that we've remained independent - we're still focused on this business. It gives us an interesting leg up in that regard. A lot of our competitors are now part of the enterprise content-management industry. And enterprise content management has no interest in what it means to work with the general consumer. They're very focused on the needs of large enterprise: things like Sarbanes - Oxley compliance, for example. Although that's very important - there has been a lack of focus on specialized markets.

I think this is an interesting opportunity for us to expand DAM into a digital-delivery environment.

MM: One last comment that I want to tag in, here. Could you speak to how North Plains is responding to the federally mandated requirement for financial reporting around the standard of XBRL?

SS: We haven't really run into that an awful lot, at this point. Again, I think we're going to, moving forward, as it's really a useful tool.

We have been heavily entrenched in the creative side of things and the statistics that we gather about assets and how they're used typically get filtered into other systems that take advantage of that information and use it specifically to create the reports and the requirements that are being mandated by that. MM: That sounds like a great place to conclude. Again, thank you so much. 